\title{
Changes and analysis of transvaginal forceps delivery rate in primary hospitals in the past 10 years
}

\author{
Liang Qiufeng, Xi Jie, Wang Xiahong, Yang Lixin \\ Shanghai Jiading District Maternity and Child Care Hospital, China
}

\begin{abstract}
Objectives: This study analyzed the changes of vaginal forceps delivery rate in Jiading Maternal and Child Health Hospital in the past 10 years in order to provide theoretical reference for reducing the rate of cesarean section and solving cephalic dystocia.

Material and methods: The basic information, delivery means and vaginal forceps indication of 78,811 parturients who gave birth in our hospital between January 1, 2009 to December 31, 2018 were analyzed retrospectively, and statistical analysis was carried out by analysis of variance and Chi-square test.

Results: In the past 10 years, there was a significant difference in the rate of vaginal forceps use among different years $(p<0.05)$. With 2014 as the turning point, the rate of forceps use increased the fastest, from $0.7 \%$ in 2013 to $3.3 \%$ in 2016. The main indications of forceps increased use in our hospital from high to low were fetal distress, abnormal occipital position, prolongation of the second stage of labor and shortening of the second stage of labor. And there was significant difference among different years $(p<0.000)$. Although there was no significant difference among the years of labor forceps use in patients with prolonged second stage of labor and abnormal occipital position $(p>0.05)$, the proportion of forceps delivery in the second stage of labor was gradually decreased with 2014 as the dividing line. Although there was significant difference among the patients who shortened the second stage of labor $\left(X^{2}=23,886, p<0.01\right)$, it ranked fourth all the time.

Conclusions: In the past 10 years, the rate of forceps use has been on the rise. With the implementation of the new stage of labor and painless delivery in 2014, vaginal forceps have become the main means to solve the problem of cephalic dystocia.

Key words: vaginal forceps; low forceps; forceps rate
\end{abstract}

Ginekologia Polska 2019; 90, 12: 711-716

\section{INTRODUCUION}

Forceps is an obstetrical operation in which the fetal head is fixed with traction or rotation force to correct the position of the fetal head and assist the descent of the fetal head and the delivery of the fetus [1].

In recent years, with the full opening of the two-child policy in China, the increase in the number of elderly and high-risk parturients, as well as the extremely high requirements for maternal and infant safety and the intervention of more social factors in medical behavior in recent years, the rate of cesarean section has been rising. The forceps rate had been declining and even abandoned for a time [2]. Although cesarean sections have solved part of the dystocia, it is a non-physiological way of delivery. After the cesarean section rate rises to a certain height, the perinatal mortality rate does not continue to decline, and it is easy to cause a variety of short-term and long-term complications $[3,4]$, especially for those who have reached $S \geq+2$ in the bone part of the fetus. Forced cesarean sections are more harmful to mothers and infants, and it is easy to cause adverse maternal and fetal outcomes.

The outcome of transvaginal forceps is closely related to the height of the fetal head. According to the maximum transverse diameter of the fetal head and the position of the lowest part of bone in the pelvis, the forceps are divided into outlet forceps, low forceps, median forceps and high forceps [5]. Because the middle and high forceps are more harmful to the mother and infant, they have been replaced by cesarean sections, with the us of low forceps, which have the advantages of convenient placement and less damage to mother and infant.Thus, they are one of the most effective means of obstetrics [6].

Our hospital has been a implementer of transvaginal low or export forceps throughout the past 10 years. Through the retrospective analysis of the changes of forceps rate, the purpose of this paper is to provide theoretical reference for 
solving cephalic dystocia, as well as, to provide theoretical reference for solving cephalic dystocia.

\section{Objectives}

The aim of this study was to provide theoretical reference for solving cephalic dystocia by analyzing the causes of the change of vaginal forceps use rate in our hospital throughout the past 10 years.

\section{MATERIAL AND METHODS \\ Participants}

With the help of the medical record management system of our hospital, the relevant information and delivery methods of 78,811 parturients who gave birth in our hospital from January 1, 2009 to December 31, 2018 were collected.

\section{Methods of data collection}

Our hospital adopts the registration book of vaginal delivery and cesarean sections made by the National Health and Family Planning Commission, which is implemented by a fixed person. The data used in this study were collected from a register book, which included maternal age, gestational weeks of delivery, delivery means, history of delivery, amount of postpartum hemorrhage and fetal weight. Also if the delivery means was forceps, the indication of forceps use should also be recorded.

\section{Forceps operator}

All cases of forceps use were assisted by Simpson or Killand forceps, and all of them were operated according to the standard of forceps by the chief resident and above professional person.

\section{Classification and description of forceps indications [7-12]}

a) The second stage of labor is prolonged: Primary parturient, the second stage of labor without epidural analgesia has been more than 3 hours, or the second stage of labor for epidural analgesia more than 4 hours. Parturients, the second stage of labor without epidural analgesia was more than 2 hours, or the second stage of labor with epidural analgesia was more than 3 hours;

b) fetal distress;

c) abnormal occipital position;

d) maternal factors need to shorten the second stage of labor: Such as pregnancy complicated with heart disease, hypertensive disorder complicating pregnancy, history of cesarean section, uterine scar etc., are not suitable to labor and abdominal pressure during delivery.

If there are two or more indications before forceps use, the first indication is included and calculated.

\section{Statistical analysis}

The data were statistically analyzed by SPSS17.0. The measurement data were expressed by $x \pm s$, the one-way ANOVA was used for comparison between groups, the frequency and rate were used for counting data, and $X^{2}$ test was used for comparison between groups. The difference was statistically significant at $p<0.05$.

\section{RESULTS}

Comparison of the general situation of the parturients overall

In 78,811 parturients, the average age was $(26.2 \pm 4.3)$ years, the average gestational weeks of the first examination was $(18.5 \pm 1.7)$ weeks, the average gestational weeks of delivery was (39.2 \pm 1.6$)$ weeks, the average amount of postpartum hemorrhage was $(219.1 \pm 86.4) \mathrm{mL}$ the average neonatal weight was $(3328.1 \pm 431.7)$ grams. There were 25,955 parturients (32.9\%), 4111 elderly parturients ( $\geq 35$ years old, 5.2\%), 4704 premature births (6.0\%), 45,586 cases $(57.8 \%)$ of transvaginal delivery, 33,225 cases $(42.2 \%)$ of cesarean sections and 1382 cases $(1.8 \%)$ of transvaginal forceps in totality. It was found in age, first gestational antenatal examination week, gestational week of delivery, postpartum hemorrhage, neonatal weight and proportion of multipara, proportion of elderly parturients, premature delivery rate, vaginal delivery rate, cesarean section rate, forceps delivery rate by comparing the general situation of the overall maternity over a 10-year period, the average value and proportion not only increase with the increase of the year, there were significant differences in every year $(p<0.01)$. See Table 1.

\section{Comparison of general situation between natural delivery and forceps}

In 45,586 cases of natural labor in the past 10 years, the average age of parturients was $(25.8 \pm 4.1)$ years, the average gestational weeks of the first examination was (16.7 \pm 6.8 ) weeks, the average gestational weeks of delivery was (39.3 \pm 1.7$)$ weeks, and the average amount of postpartum hemorrhage was $(231.3 \pm 95.1) \mathrm{mL}$, and the average neonatal weight was (3344.9 \pm 428.6$)$ grams. There were 15,950 cases (35\%) of multiparas, 2309 cases $(5.1 \%)$ of elderly parturients ( $\geq 35$ years old), 220 cases of premature delivery $(0.5 \%)$. the average length of second stage of labor was $(116 \pm 39)$ minutes and 25528 cases of epidural analgesia (56.0\%).

In 1382 parturients with forceps use over the past 10 years, the average age was $(27.7 \pm 4.2)$ years, the average gestational weeks of the first examination was (15.4 \pm 4.9$)$ weeks, the average gestational weeks of delivery was $(39.0 \pm 1.7)$ weeks, and the average amount of postpartum hemorrhage was $(389.0 \pm 98.5) \mathrm{mL}$. The average neonatal 


\begin{tabular}{|c|c|c|c|c|c|c|c|}
\hline Year & Num. & Age (year) & FAE (week) & GWD (week) & PPH (mL) & \multicolumn{2}{|l|}{ NW (gram) } \\
\hline 2009 & 6881 & $24.6 \pm 4.1$ & $21.7 \pm 8.0$ & $39.3 \pm 1.7$ & $200.8 \pm 81.9$ & \multicolumn{2}{|c|}{$3299.6 \pm 450.3$} \\
\hline 2010 & 7217 & $25.1 \pm 3.8$ & $20.8 \pm 7.1$ & $39.0 \pm 1.5$ & $198.6 \pm 91.6$ & \multicolumn{2}{|c|}{$3303.5 \pm 431.2$} \\
\hline 2011 & 7540 & $24.9 \pm 4.9$ & $21.1 \pm 3.2$ & $39.0 \pm 1.7$ & $201.3 \pm 78.5$ & \multicolumn{2}{|c|}{$3298.6 \pm 432.7$} \\
\hline 2012 & 8064 & $25.2 \pm 4.4$ & $20.8 \pm 8.2$ & $39.1 \pm 1.8$ & $205.7 \pm 79.8$ & \multicolumn{2}{|c|}{$3305.5 \pm 445.2$} \\
\hline 2013 & 7346 & $25.8 \pm 4.5$ & $20.6 \pm 7.9$ & $39.1 \pm 1.6$ & $203.2 \pm 83.6$ & \multicolumn{2}{|c|}{$3310.1 \pm 420.1$} \\
\hline 2014 & 8561 & $26.4 \pm 4.4$ & $17.8 \pm 8.4$ & $39.2 \pm 1.7$ & $221.4 \pm 76.8$ & \multicolumn{2}{|c|}{$3328.4 \pm 435.4$} \\
\hline 2015 & 6920 & $26.8 \pm 4.3$ & $17.2 \pm 6.6$ & $39.1 \pm 1.5$ & $225.7 \pm 82.9$ & \multicolumn{2}{|c|}{$3352.4 \pm 432.5$} \\
\hline 2016 & 9329 & $27.4 \pm 4.2$ & $15.6 \pm 6.1$ & $38.9 \pm 1.9$ & $227.0 \pm 99.9$ & \multicolumn{2}{|c|}{$3348.2 \pm 435.5$} \\
\hline 2017 & 8798 & $27.9 \pm 4.3$ & $14.7 \pm 5.3$ & $39.0 \pm 1.8$ & $244.7 \pm 90.1$ & \multicolumn{2}{|c|}{$3357.7 \pm 422.8$} \\
\hline 2018 & 8491 & $28.3 \pm 4.5$ & $14.8 \pm 5.0$ & $39.1 \pm 1.3$ & $262.9 \pm 98.6$ & \multicolumn{2}{|c|}{$3376.6 \pm 411.9$} \\
\hline$F$ & & 182,399 & 421,306 & 6.589 & 145,489 & \multicolumn{2}{|l|}{10.444} \\
\hline$P$ & & 0.000 & 0.000 & 0.000 & 0.000 & \multicolumn{2}{|l|}{0.000} \\
\hline Year & Num. & Mul. (\%) & $\geq 35 Y(\%)$ & PD (\%) & VD (\%) & Force (\%) & CS (\%) \\
\hline 2009 & 6881 & $1933(28.1)$ & $241(3.5)$ & $399(5.8)$ & $4004(58.2)$ & $70(1.0)$ & $2877(41.8)$ \\
\hline 2010 & 7217 & $2165(30.0)$ & $282(3.9)$ & $426(5.9)$ & $4115(57.0)$ & $60(0.8)$ & $3102(43.0)$ \\
\hline 2011 & 7540 & $2189(29.0)$ & $309(4.1)$ & $430(5.7)$ & $4261(56.5)$ & $65(0.9)$ & $3279(43.5)$ \\
\hline 2012 & 8064 & $2452(30.4)$ & $323(4.0)$ & $427(5.3)$ & $4230(52.5)$ & $67(0.8)$ & $3834(47.5)$ \\
\hline 2013 & 7346 & $2283(31.1)$ & $305(4.2)$ & $386(5.3)$ & $3788(51.5)$ & $54(0.7)$ & $3558(48.4)$ \\
\hline 2014 & 8561 & $2915(34.0)$ & $359(4.2)$ & $514(6.0)$ & $4936(57.7)$ & $90(1.1)$ & $3625(42.3)$ \\
\hline 2015 & 6920 & 2288 (33.1) & $352(5.1)$ & $436(6.3)$ & 4139 (59.8) & $160(2.3)$ & $2781(40.2)$ \\
\hline 2016 & 9329 & $3171(34.0)$ & $513(5.5)$ & $587(6.3)$ & $5633(60.4)$ & $306(3.3)$ & $3696(39.6)$ \\
\hline 2017 & 8789 & 3333 (37.9) & $655(7.5)$ & $546(6.2)$ & $5392(61.3)$ & $258(2.9)$ & $3397(38.7)$ \\
\hline 2018 & 8164 & 3226 (39.5) & $772(9.5)$ & $553(6.8)$ & $5088(62.3)$ & $252(3.1)$ & $3076(37.7)$ \\
\hline$x^{2}$ & & 456,283 & 531,283 & 28.024 & 369,806 & 494,855 & 369,806 \\
\hline$P$ & & 0.000 & 0.000 & 0.000 & 0.000 & 0.000 & 0.000 \\
\hline
\end{tabular}

$\mathrm{PPH}$ - postpartum hemorrhage; FAE — first antenatal examination; GWD — gestation week of delivery; NW — neonatal weight; Mul. — Multipara; PD — premature delivery; VD - vaginal delivery; $C S$ - caesarean section; $\geq 35 \mathrm{Y}-\geq 35$ years old of parturients

weight was (3434.2 \pm 457.5$)$ grams, There were 161 cases (11.6\%) of multiparas, 72 cases $(5.2 \%)$ of elderly parturients ( $\geq 35$ years old), 19 cases of premature delivery (1.4) the average length of second stage of labor was (139 \pm 53 ) minutes and 805 cases of epidural analgesia (56.0\%).

It was found that there were significant differences in age, gestational week, postpartum hemorrhage, neonatal weight and the proportion of multiparas $(p<0.05)$ by comparing the general situation of the two groups. However, there was no significant difference in gestation week of delivery, the length of the second stage of labor and the multiparas and the proportion of epidural analgesia deliver $(p>0.05)$.

See Table 2.

\section{Change of forceps delivery rates}

Troughout the past 10 years, among the 78811 parturients, there were 45,586 cases of natural delivery, 33,225 cases of cesarean section and 1382 cases of forceps. The rate of forceps delivery in total delivery was 1.8\% (1382/78811).
The proportion of forceps delivery in natural delivery was 2.9\% (1382-46968). The proportion of forceps use increased with the increase of the year, and the rate of forceps delivery increased the fastest with the turning point of 2014, it from 0.7\% in 2013 to 3.3\% in 2016, as shown in Figure 1.

\section{Comparison of forceps delivery indications}

In the past 10 years, the main indications of forceps use in our hospital from high to low were fetal distress, abnormal occipital position, prolongation of the second stage of labor and shortening of the second stage of labor. The proportion of forceps use in fetal distress increased year by year, from third to first place, and there was significant difference among different years $\left(X^{2}=32,077, p<0.05\right)$. Although the proportion of labor forceps use prolonged in the second stage of labor gradually decreased from the first to the third, there was no significant difference between the two groups $(p>0.05)$.There was no significant change in the proportion of forceps delivery due to abnormal occipital position 
Table 2.Comparison of general situation between natural delivery and forceps

\begin{tabular}{|l|l|l|l|l|l|l|}
\hline Group & Num. & Year & FAE (week) & GWD (week) & PPH (mL) & NW (g) \\
\hline Nat. & 45586 & $25.8 \pm 4.1$ & $16.7 \pm 6.8$ & $39.3 \pm 1.7$ & $231.3 \pm 95.1$ & $3344.9 \pm 428.6$ \\
\hline For. & 1382 & $27.7 \pm 4.2$ & $15.4 \pm 4.9$ & $39.0 \pm 1.7$ & $389.0 \pm 98.5$ & $3434.2 \pm 457.5$ \\
\hline F & & 90.208 & 76.004 & 1.081 & 274,076 & 4.711 \\
\hline P & & 0.000 & 0.000 & 0.299 & 0.000 & 0.030 \\
\hline
\end{tabular}

\begin{tabular}{|l|l|l|l|l|l|l|}
\hline Group & Num. & Mul. (\%) & $\geq 35 Y(\%)$ & PD (\%) & LOSSOL (min.) & EA (\%) \\
\hline Nat. & 45586 & $15950(35.0)$ & $2309(5.1)$ & $220(0.5)$ & $116 \pm 39$ & $25528(56.0)$ \\
\hline For. & 1382 & $161(11.6)$ & $72(5.2)$ & $19(1.4)$ & $139 \pm 53$ & $805(58.2)$ \\
\hline F & & 324,000 & 0.058 & 21.091 & 0.788 & 2.755 \\
\hline P & & 0.000 & 0.809 & 0.000 & 0.395 & 0.097 \\
\hline
\end{tabular}

LOSSOL - length of second stage of labor; EA — epidural analgesia

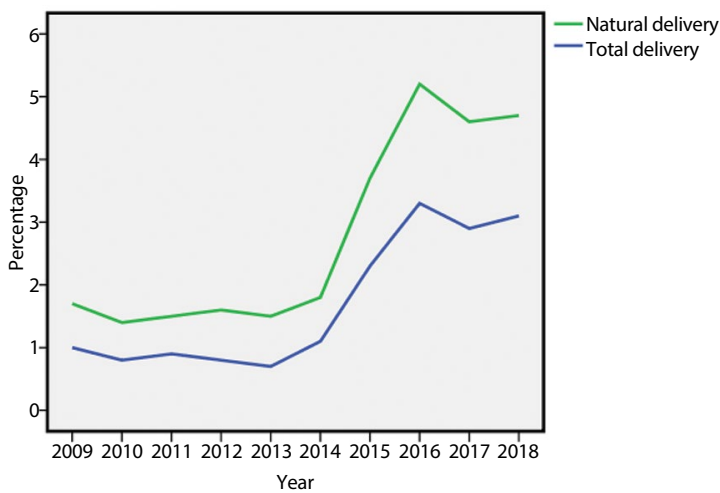

Figure 1. Trends in the proportion of forceps in total and natural delivery

and other reasons, and there was no significant difference between the two groups ( $p>0.05$ ). The shortening of the second stage of labor was always in the fourth place, and there was significant difference between the two groups $\left(X^{2}=23,886, P<0.01\right)$. See Table 3 .

\section{CONCLUSIONS}

It is reviewed that vaginal forceps delivery has a history of more than 200 years, and it is still an indispensable means in the treatment of dystocia. Previous studies have shown that the current clinical use of low birth forceps can not only effectively reduce the rate of cesarean section [13], but also a main means to solve cephalic dystocia in addition to cesarean section [14]. The following is a brief analysis of the situation of forceps delivery in our hospital in the past 10 years.

\section{Analysis of the overall situation of parturients}

Over10 years it was found that there were significant difference among the groups in age, first antenatal examination week, postpartum hemorrhage, neonatal weight and

\begin{tabular}{|c|c|c|c|c|c|c|}
\hline Ind. & Forceps & FD & AOP & LOSSOL & SCSOL & Others \\
\hline 2009 & 70 & $16(22.8)$ & $20(28.6)$ & 25 (35.7) & $7(10.0)$ & $2(2.9)$ \\
\hline 2010 & 60 & $14(23.3)$ & $17(28.3)$ & $20(33.3)$ & $9(15.0)$ & 0 \\
\hline 2011 & 65 & $16(24.6)$ & 18 (27.7) & $23(35.4)$ & $7(10.8)$ & $1(1.5)$ \\
\hline 2012 & 67 & $15(22.4)$ & $20(29.9)$ & $24(35.8)$ & $6(9.0)$ & $2(3.0)$ \\
\hline 2013 & 54 & $15(27.8)$ & $16(29.6)$ & $18(33.3)$ & $6(11.1)$ & 0 \\
\hline 2014 & 90 & $27(30.0)$ & $28(31.1)$ & $29(32.2)$ & $4(4.4)$ & $1(1.1)$ \\
\hline 2015 & 160 & $52(32.5)$ & $48(30.0)$ & $45(28.3)$ & $12(7.5)$ & $3(1.9)$ \\
\hline 2016 & 306 & $112(36.6)$ & $88(28.9)$ & $89(29.8)$ & $15(4.9)$ & $2(0.7)$ \\
\hline 2017 & 258 & $102(39.5)$ & 75 (29.7) & 72 (27.9) & $7(2.7)$ & $2(0.8)$ \\
\hline 2018 & 252 & $113(44.8)$ & $70(27.8)$ & $60(23.8)$ & $9(3.6)$ & 0 \\
\hline$x^{2}$ & & 32.007 & 0.570 & 9.215 & 23.886 & 10.998 \\
\hline$P$ & & 0.000 & 1.000 & 0.418 & 0.004 & 0.121 \\
\hline
\end{tabular}

Ind. — indication; SCSO — shorten the second stage of labor; FD — fetal distress; AOP — abnormal occipital position; LOSSOL — length of second stage of labor 
proportion of multiparas, proportion of elderly parturients, premature delivery rate, vaginal delivery rate, cesarean section rate and forceps rate by comparing 78,811 parturients. It was also found that the average age of mothers was increasing, from 24.6 years in 2009 to 28.3 years in 2018. During this period, the elderly and parturients were also increasing accordingly, which were positively correlated with the postponement of the age of marriage and childbearing in China in recent years. However, the first Gestational examination week decreased from 21.7 weeks in 2009 to 14.8 weeks in 2018 , which was related to the importance of antenatal care and the enhancement of maternal health awareness in China. Although the gestational weeks and premature delivery rates were different in each year, the overall increase was not obvious. the gestational weeks were about 39 weeks, and the premature delivery rate increased slightly, fluctuating between $5 \%$ and $7 \%$. At the same time, it was also found that with the increase of neonatal weight, the amount of postpartum hemorrhage was also increasing, from the average $200.8 \mathrm{~mL}$ in 2009 to $262.9 \mathrm{~mL}$ in 2018, which fully proved that the higher the fetal weight, the higher the incidence of postpartum hemorrhage.

\section{Analysis of natural and forceps delivery}

In the past 10 years, there were 45,586 cases of natural delivery and 1382 cases of forceps. After comparing the two groups of data, it was found that there was no significant difference in the elderly. However, the average age were 27.7 years of forceps delivery, while the average age were 25.8 years of natural delivery, and the proportion of parturients in forceps was $11.6 \%$, which was significantly lower than that of $35 \%$ in the natural delivery group, which indicated that the primipara with relatively older age, the higher the dystocia rate, and the greater the risk of deliver, it is considered to be related to the decrease of physical strength and ligament elasticity. In addition, there were significant differences in the first gestational age, postpartum hemorrhage, premature delivery rate and neonatal weight between the two groups. Although it has been shown that fetal weight is proportional to the incidence of postpartum hemorrhage, the neonatal average weight were $3434 \mathrm{~g}$ in the forceps group which is slightly higher than in the natural delivery whose average weight were 3344 g.The difference in the amount of postpartum hemorrhage was the largest which with an average of $231 \mathrm{~mL}$ in the natural delivery group and $389 \mathrm{~mL}$ in the forceps delivery group. The analysis of postpartum hemorrhage is often complicated, which is not only caused by a single factor. In this study, the average time was 139 minutes of the second stage of labor in the forceps group was longer than that in the natural group whose was 116 minutes, which also increases the incidence of postpartum hemorrhage.

\section{Forceps delivery rate and its trends}

The rate of forceps use varies greatly in the world, which depends on the medical resources and clinical training mode. The proportion of forceps midwifery in natural delivery in the United States is $0.56 \%$ [7]. Due to the regional economic differences and the uneven development of medical level, the utilization rate of forceps varies greatly in hospitals at all levels, so there are different reports in different regions and hospitals. In this study, the rate of forceps use in the total delivery in our hospital in the past 10 years was $1.8 \%$, and the proportion of forceps used in vaginal delivery was $2.9 \%$ (1382/46968), which was higher than that in the United States reported in the literature. In recent years, the rate of forceps use in our hospital has been increasing, especially in 2014 as a turning point. Under the background of the implementation of the new stage of labor and the painless delivery in our hospital, it is easier to implement forceps.

At this time, our hospital also strengthened the personnel training of forceps and began to carry out forceps from the chief resident, so that the minimum birth forceps delivery rate increased from 2013 to 3.3 percent in 2016. at the same time, the rate of cesarean section also decreased accordingly. The rate of cesarean section decreased from $48.5 \%$ in 2013 to $37.7 \%$ in 2018, which proved that forceps not only solved cephalic dystocia, but also effectively reduced the rate of cesarean section.

\section{Comparison of indications of forceps}

Over the past 10 years, the main indications of forceps use in our hospital from high to low were fetal distress, occipital abnormality, prolongation of the second stage of labor and shortening of the second stage of labor, which was consistent with the indication of forceps use. Comparing the indications of forceps use in each year, it was found that the proportion of forceps use in fetal distress was gradually higher, from the third to the first, and the difference was statistically significant. This is mainly considered with the implementation and strengthening of continuous fetal electronic monitoring, It's also becoming more and more likely to find fetal distress clinically, therefore, the proportion of forceps use due to fetal distress is gradually increasing. In addition, although there was no significant change in the proportion of forceps use in patients with prolonged second stage of labor and abnormal occipital position, considering that since the implementation of the new stage of labor in China in 2014 and the increase in the number of epidural anesthesia in recent years, the proportion of clinically diagnosed prolonged second stage of labor has decreased, and the proportion of forceps performed accordingly is decreasing. It fell from $35.7 \%$ in 2009 to $23.8 \%$ in 2018 . In order to shorten the second stage of labor, there was a significant 
difference among the years, but it was always in the fourth place. Considering the full development of painless delivery, the second stage of labor was prolonged accordingly. On the other hand, the number of forceps with the indication of shortening the second stage of labor is decreasing accordingly. As for other indications, because the number of cases is too few, the proportion is relatively low, and there is no statistical difference among the groups, the analysis is not done here.

\section{Advantages and disadvantages of this study}

Although this study spans 10 years and has a large number of cases, it is only a retrospective study which has some limitations. Only the changes of the general situation, forceps use rate and indication of parturients were analyzed, but the birth injury caused by forceps and the detailed pregnancy outcome were not reflected in this study. In addition, the data of this study only comes from a second-class specialized hospital, the research object has limitations, but also affects the promotion and extension of the research results.

In short, with the opening of the "comprehensive two-child policy" in China, the number of elderly parturients and pregnant women with scar uterus has increased, the demand for cesarean section is also increasing, but with the implementation of the new stage of labor and the development of painless delivery, low forceps has become the main means of delivery to solve cephalic dystocia.

\section{Funding}

Research was supported by Jiading key medical specialty funding (funding code: JDXYZDZK-008).

\section{Acknowledgements}

The author would like to thank for the persons who checks medical history and records data. We would also thank Professor Zhu Changtai's encouragement and guidance.

\section{REFERENCES}

1. Yu Xinshuan, Qi Hongbo. Low forceps midwifery [J/CD]. Chinese Journal of Obstetric first Aid. 2018; 7(3): 144-147.

2. Zhu Lin, Zhang Xiaoyan, Bao Di, etc. The significance of forceps in reducing the rate of cesarean section [J]. Armed Police Medicine. 2015; 26(12): 1196-1198.

3. Ren Nianzhen. Clinical analysis of 119 cases of cesarean section forceps [J]. Forum on Primary Medicine. 2015; 19(8): 1150-1151.

4. Qi Huiyi, Wang Liaoju. Clinical analysis of 48 cases of caesarean section forceps [J]. Chinese Maternal and Child Health Research. 2010; 21(3): 66-367.

5. Committee on Practice Bulletins-Obstetrics.ACOG Practice Bulletin No. 154: Operative vaginal delivery [J]. Obstet Gynecol. 2015; 126(5): e56-e65.

6. Rossignol M, Chaillet N, Boughrassa F, et al. Interrelations between four antepartum obstetric interventions and cesarean delivery in women at low risk: a systematic review and modeling of the cascade of interventions. Birth. 2014; 41(1): 70-78, doi: 10.1111/birt.12088, indexed in Pubmed: 24654639.

7. Practice Bulletin No. 154. Obstetrics \& Gynecology. 2015; 126(5): e56-e65, doi: 10.1097/aog.0000000000001147.

8. American College of Obstetrics and Gynecology.. Operative vaginal delivery. Clinical management guidelines for obstetrician-gynecologists. American College of Obstetrics and Gynecology. Int J Gynaecol Obstet. 2001;74(1):69-76, doi: 10.1016/s0020-7292(01)00434-9, indexed in Pubmed: 11480404.

9. Royal College of Obstetricians and Gynaecologists (RCOG). Operative

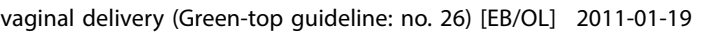
[2016-06-02] https://www rcog org uk/en/guidelines-research-services/guidelines/gtg26.

10. Cargill YM, MacKinnon CJ, Cargill YM, et al. Clinical Practice Obstetrics Committee. Guidelines for operative vaginal birth. J Obstet Gynaecol Can. 2004; 26(8): 747-761, doi: 10.1016/s1701-2163(16)30647-8, indexed in Pubmed: 15307980.

11. Obstetrics and Gynecology Credit Association of Chinese Medical Association obstetrics and Gynecology Section. Expert consensus on new labor process standards and handling. Chinese Journal of Obstetrics and Gynecology. 2014; 49(7): 486, doi: 10.3760/cma.j.issn.0529-56 7x.2014.07.002.

12. Obstetric Care Consensus No. 1. Obstetrics \& Gynecology. 2014; 123(3): 693-711, doi: 10.1097/01.aog.0000444441.04111.1d.

13. Moreau R, Pham MT, Brun X, et al. Assessment of forceps use in obstetrics during a simulated childbirth. Int J Med Robot. 2008; 4(4): 373-380, doi: 10.1002/rcs.222, indexed in Pubmed: 19006201.

14. Yang Lu, Tan Lizhen. Clinical analysis of 120 cases of low forceps [J]. Clinical Medical Engineering. 2017; 24(1): 71. 